\title{
Melanoma TRP2 CTL Epitope Vaccine SCIB1
}

National Cancer Institute

\section{Source}

National Cancer Institute. Melanoma TRP2 CTL Epitope Vaccine SCIB1. NCI Thesaurus.

Code C91380.

A proprietary DNA-based cancer vaccine that encodes a melanoma antigen tyrosinaserelated protein 2 (TRP2) cytotoxic T-lymphocyte (CTL) epitope and a modified monoclonal antibody, a chimera of human IgG1/murine Ig G2a with $T$ cell mimotopes expressed within the complementarity-determining regions (CDR) of the antibodies, with potential immunostimulating and antineoplastic activities. Upon intramuscular injection and electroporation, melanoma TRP2 CTL epitope vaccine SCIB1 expresses the modified antibody. Subsequently, the Fc component of the eng ineered antibody targets and binds to the CD64 receptor on the dendritic cells (DCs); upon processing by DCs, the cellular immune system may be activated to induce helper $\mathrm{T}$-cell and $\mathrm{CT} L$ immune responses against tumor cells expressing the TRP2 antigen. 